\title{
ACTITUDES HACIA LAS PERSONAS CON DISCAPACIDAD DE ESTUDIANTES DE LA UNIVERSIDAD DE GRANADA
}

\author{
ATTITUDES OF UNIVERSITY STUDENTS FROM GRANADA \\ TOWARDS PEOPLE WITH DISABILITIES
}

\author{
María Tamara Polo Sánchez* y María Dolores López Justicia**
}

\begin{abstract}
RESUMEN
Este artículo recoge información sobre las actitudes que presentan los estudiantes universitarios granadinos hacia la discapacidad. La escala que se utilizó para medir estas actitudes fue la Escala de Actitudes hacia las personas con discapacidad (Verdugo, Jenaro y Arias, 1995). Se aplicó a un grupo de 488 estudiantes universitarios de distintas titulaciones de Magisterio (Audición y Lenguaje, Educación Especial, Educación Musical, Educación Física), Licenciatura en Psicopedagogía, Psicología y Educación Física. Los resultados mostraron que los estudiantes presentan actitudes positivas hacia los discapacitados, lo que puede deberse a la competencia de sus compañeros con discapacidad, a las acciones emprendidas por los servicios de orientación o a otras razones pendientes de estudio.
\end{abstract}

Palabras clave: Estudiantes universitarios - Discapacidad - Actitudes.

\begin{abstract}
This paper describes a study of university student's attitudes towards people with disabilities. One scale measured subject's attitudes towards people with disabilities, Actitudes hacia las personas con discapacidad (Verdugo, Jenaro y Arias, 1995). This scale were administered to 487 university students from Magisterio (Audición y Lenguaje, Magisterio de Educación Especial, Magisterio de Educación Musical, Magisterio de Educación Física), Licenciatura en Psicopedagogía, Psicología y Educación Física. The results showed that students have positive attitudes towards people with disabi-
\end{abstract}

* $\quad M^{a}$ Dolores López Justicia es Profesora titular del Departamento de Psicología Evolutiva y de la Educación de la Universidad de Granada, entre sus líneas de investigación se encuentran: Inserción laboral de estudiantes universitarios/as con discapacidad física y sensorial. Desarrollo socio-personal de afectados/as por Retinosis Pigmentaria. Autoconcepto de niños, adolescentes y adultos afectados por baja visión. Desarrollo socio-personal de personas con afectación sensorial o motora. Correo-e: dlopezj@ugr.es

** $\quad M^{a}$ Tamara Polo Sánchez es Licenciada en Psicopedagogía y estudiante del programa de doctorado "La Educación: Problemas y Soluciones Psicoeducativas". Correo-e: tpolo@ugr.es 
lities which can be due to the competence of their colleagues with disabilities, to the actions undertaken by the services of counseling or to other pending reasons of study.

Keywords: University students - Disabilities - Attitudes.

\section{Introducción}

El avance que se ha ido produciendo en los últimos años en la atención a las personas con discapacidad ha hecho posible su incorporación a la enseñanza en todos los niveles. La enseñanza universitaria no ha sido una excepción en este avance, afortunadamente, de modo que actualmente encontramos cada vez más a menudo en los centros universitarios españoles, al igual que en el resto del mundo occidental, personas que presentan alguna discapacidad. De acuerdo con Alcantud (1995), los tipos de discapacidades que con mayor frecuencia padecen los estudiantes españoles al acceder a la Universidad son: deficiencia sensorial (auditiva y visual), deficiencia motora y de desplazamiento, enfermedades de larga duración y/o especial tratamiento (Alcantud, 1995). Los datos recogidos por el Gabinete de Atención Social de la Universidad de Granada coinciden con estas categorías, pues durante el curso académico 2003-2004 se matricularon en la misma 287 alumnos que presentaban alguna de esta discapacidad. Más detalladamente: un 58,9\% (169) de los alumnos matriculados estaban afectados de discapacidad física, un 13,6\% (39) de discapacidad sensorial auditiva, un 17,1\% (49) presentaba discapacidad sensorial visual, y un 3,5\% (10) déficit psíquico.

Si bien se acrecienta cada vez más la presencia de discapacitados en la universidad, este porcentaje es todavía bajo. Las explicaciones a este hecho, que a su vez también se manifiestan en otros niveles educativos, son muy diversas y tienen relación con el accidentado itinerario educativo que desarrollan la mayoría de las personas con discapacidad y la ruptura que se produce entre la atención que estos alumnos reciben en los niveles educativos obligatorios y los no obligatorios.

Los alumnos con discapacidad se enfrentan en ocasiones a determinadas situaciones que entorpecen el acceso a los estudios universitarios con normalidad, pues la discapacidad puede repercutir en el ámbito académico, en el campo de las relaciones y en el contexto de la vida cotidiana (López y Chacon, 2004). Asimismo, el acceso a la universidad y a los propios estudios universitarios están plagados de procesos de selección y de filtros que no suelen tener en cuenta las posibilidades físicas o sensoriales del alumno (Alcantud, 1995; Figuera, 1996). Entre los obstáculos que pueden encontrarse se señalan los siguientes:

- Nivel de autoestima del alumno con discapacidad durante el proceso educativo.

- Las barreras arquitectónicas (así como el resto de factores contextuales), de transporte y de acceso a los centros educativos.

Lo expuesto da una idea aproximada de que el acceso de las personas con discapacidad a la universidad no es una tarea fácil y, por si fuera poco, además, pueden encontrar otras barreras: las referidas a las actitudes generadas hacia las personas discapacitadas, o la percepción que se tiene de la discapacidad. Se trata de barreras de tipo psicológico que se pueden configurar en las actitudes de los compañeros, profesores, familia y sociedad.

El tema de las actitudes sociales hacia las personas con discapacidad es de gran relevancia porque muchas veces inciden de una u otra forma en las posibilidades de integración so- 
cial de las personas con discapacidad, llegando a transformarse en una variable decisiva (Larrivee, 1982).

Según Triandis (1971), la actitud es una idea cargada de emoción que predispone a una clase de acciones ante un determinado tipo de situaciones sociales, de la que se pueden desprender tres componentes: la emoción, o componente afectivo; la idea, o componente cognitivo; y un componente conductual, o predisposición a la acción.

El estudio de las actitudes hacia la discapacidad se ha situado en torno a estudios diferenciados en función del colectivo al que se ha dirigido (Verdugo, Jenaro y Arias, 1995); fundamentalmente se han analizado las actitudes de los maestros y profesionales de la enseñanza (Alemany, 2003; Rizzo y Wright, 1988), las actitudes de padres y familiares (Zulueta, 1991) y las actitudes de los compañeros/iguales universitarios hacia los discapacitados (Gómez e Infante, 2004; González, Gilar y Pérez, 2002).

Los estudios centrados en la evaluación de las actitudes de maestros se han hecho desde diferentes perspectivas, entre las que destacan la centrada en la percepción de la competencia como factor clave que condiciona una actitud positiva (Rizzo y Wright, 1988). Desde esta vertiente aquellos docentes que tienen un adecuado entendimiento de la naturaleza de la excepcionalidad y un conocimiento sobre las técnicas y métodos instruccionales están más capacitados para la enseñanza de los niños excepcionales, favoreciendo el desarrollo de un contexto de aceptación.

Es preciso mencionar, en esta dirección el estudio realizado por Alemany (2003) sobre las actitudes del profesorado hacia la integración del alumnado con necesidades educativas especiales. Entre los resultados obtenidos destaca que los docentes, en general, consideraron que hay que cambiar su forma de trabajar en el aula de integración. En este estudio se ponía de relieve que los maestros manifestaban falta de tiempo, de preparación, de recursos y de medios para enfrentarse a la educación de un alumno con discapacidad. Al mismo tiempo, afirmaban la existencia de una despreocupación de la Administración Educativa por este tema, dejando al tutor del aula la responsabilidad del alumnado con necesidades educativas especiales.

En cuanto a la evaluación de las actitudes de padres y familiares de personas con discapacidad, se ha investigado poco este terreno. En España existen muy pocos estudios concluyentes sobre las actitudes de los padres hacia hijos discapacitados y, generalmente, suelen hacer referencia al proceso de adaptación ante el nacimiento de un hijo con discapacidad, indicando la existencia de fases comunes a todos los padres con hijos discapacitados (Zulueta, 1991).

Gómez e Infante (2004) recogieron información relativa a las actitudes que presentaban 297 estudiantes chilenos universitarios de la Pontificia Universidad Católica de Chile hacia las personas con discapacidad y hacia la integración de las minorías étnicas. Los resultados obtenidos mostraron que todos los estudiantes presentaban actitudes positivas, siendo los alumnos de Pedagogía de último año de carrera los que mostraban actitudes significativamente más altas.

Más reciente en el tiempo, en un artículo publicado en el Diario Hoy (2/2/05) se presenta un estudio realizado por López, centrado en el análisis de las actitudes del alumnado universitario extremeño ante las personas con discapacidad. En su trabajo distinguió las actitudes positivas o negativas en función del género y titulación cursada, concluyendo que 
las estudiantes de la Universidad de Extremadura tenían una actitud más positiva ante las personas con discapacidad que sus compañeros varones; y al mismo tiempo los alumnos y alumnas procedentes de las carreras de Derecho y Medicina tenían unas actitudes menos positivas que los de otras titulaciones, incluidas las técnicas. Entre las carreras cursadas por estudiantes con actitudes más positivas destacaban las de educación.

Como puede observarse, a consecuencia de la presencia de discapacitados en la propia universidad española se han planteado distintos estudios. En esta dirección se encuentra el presente, el cual tenía como fin analizar las actitudes de estudiantes universitarios granadinos hacia las personas con discapacidad en general, pues estas actitudes pudieran ser las mismas que se sostuvieran hacia sus compañeros, o futuros compañeros, con discapacidad.

\section{Método}

La metodología de investigación seleccionada para la puesta en práctica de este trabajo fue descriptiva, basada en la aplicación de una encuesta como forma de recogida de información, ya que el principal fin era describir sistemáticamente hechos y características de una población dada, o área de interés, de forma objetiva y comprobable. Esta metodología resulta muy apropiada en este campo educativo, especialmente cuando se trabaja, como es el caso que nos ocupa, con un amplio número de sujetos en los que es difícil de acceder a la totalidad de opiniones (Colás, 1994) porque nos permite:

- Recoger información factual detallada.

- Identificar problemas (actitudes positivas y/o negativas).

- Realizar comparaciones y evaluaciones.

- Planificar futuros y tomar decisiones.

El estudio, de carácter transversal, planteado supuso la recogida de información en una muestra una sola vez y durante un período de corta duración, con el fin de captar ciertos fenómenos presentes en el momento de aplicación del instrumento de medida. El trabajo se basó en la descripción de las opiniones expresadas por los universitarios granadinos, para detectar y conocer sus actitudes hacia la discapacidad. Este tipo de metodología ha requerido el uso de un instrumento de recogida de información, con validez y fiabilidad adecuada.

\section{Población y muestra}

La elección de la muestra de estudiantes se realizó de acuerdo con las normas de representatividad, estratificando la población universitaria granadina. El primer paso consistió en seleccionar los centros universitarios en función del mayor o menor grado de presencia de estudiantes discapacitados, a continuación se seleccionaron aleatoriamente los sujetos sin discapacidad que iban a formar parte de la muestra. En la tabla 1 se representa gráficamente dicha selección muestral.

Participaron en total 488 estudiantes, de los cuales 240 eran hombres (49,2\%) y 248 mujeres $(50,8 \%)$.

En la figura 1 se puede apreciar el porcentaje de participación de acuerdo con la titulación cursada. 
TABLA 1: Distribución de alumnos universitarios por centro y titulación.

\begin{tabular}{|c|c|c|c|c|c|c|c|}
\hline Centro & \multicolumn{5}{|c|}{ Facultad de Ciencias de la Educación } & $\begin{array}{c}\text { Facultad } \\
\text { de } \\
\text { Psicología }\end{array}$ & $\begin{array}{c}\text { F.C.C.A. } \\
\text { Física y } \\
\text { Deportes }\end{array}$ \\
\hline Especialidad & $\begin{array}{c}\text { Audición } \\
\text { y } \\
\text { Lenguaje }\end{array}$ & $\begin{array}{c}\text { Educación } \\
\text { Especial }\end{array}$ & $\begin{array}{c}\text { Educación } \\
\text { Musical }\end{array}$ & $\begin{array}{c}\text { Educación } \\
\text { Física }\end{array}$ & Psicopedagogía & & \\
\hline Curso & $3^{\circ}$ & $1^{\circ}$ & $1^{\circ}$ & $1^{\circ}$ & $1^{\circ}$ & $3^{\circ}$ & $3^{\circ}$ \\
\hline $\begin{array}{c}\mathrm{N}^{\circ} \\
\text { Participantes }\end{array}$ & 60 & 50 & 40 & 138 & 29 & 24 & 147 \\
\hline
\end{tabular}

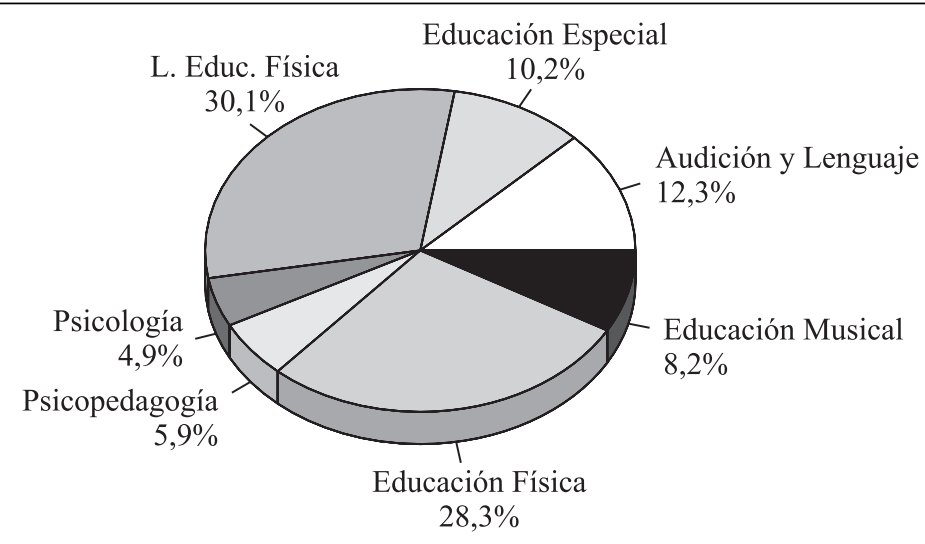

FIGURA 1.

Titulación.

Destaca una mayor participación de estudiantes, procedentes de la licenciatura en Educación Física (30,1\%), seguidos de la especialidad en Magisterio de Educación Física $(28,3 \%)$. En otro extremo se encontrarían las titulaciones de Psicología y Psicopedagogía, con una menor presencia de participantes, $4,9 \%$ y 5,9\%, respectivamente.

\section{Instrumento}

El instrumento empleado en este trabajo fue la escala de Actitudes hacia las personas con discapacidad (Verdugo, Jenaro y Arias, 1995). Este instrumento se puede utilizar, dada su fiabilidad y validez, para evaluar las actitudes en cualquier ámbito de trabajo con esta población. Los autores estimaron la fiabilidad global de la escala mediante tres métodos diferentes: el coeficiente alfa de Cronbach, dirigido a evaluar el grado de consistencia interna; el método de división en mitades con el propósito de evaluar qué punto de las dos mitades de la escala compuestas por ítems seleccionados aleatoriamente miden un único constructo; 
y el método Lambda de Guttman, cuya finalidad es examinar el nivel de fiabilidad que tendría la escala completa si ésta se dividiese en dos partes y se combinasen a continuación. El coeficiente alfa de Cronbach es .92. Los autores desarrollaron una escala multidimensional de evaluación de actitudes hacia las personas con discapacidad, partiendo de las investigaciones anteriores realizadas en España y en otros países. Para ello se seleccionaron unos ítems que fueron sometidos a juicio de expertos en la materia y posteriormente se aplicaron a un numeroso colectivo de profesionales de diversos ámbitos $(\mathrm{N}=250)$. Una vez obtenida la fiabilidad y validez de las escalas (una general y otras específicas para deficiencias físicas, sensoriales y mentales) se cuenta con un instrumento útil, desarrollado en España y con las suficientes garantías psicométricas.

La escala, tras una primera revisión, está compuesta por 37 ítems.

La tarea que debe realizar la persona evaluada consiste en opinar si está de acuerdo o no con cada una de las frases que se presentan, siendo los significados de las opiniones los siguientes:
MA Estoy muy de acuerdo
BA Estoy bastante de acuerdo
PA Estoy parcialmente de acuerdo
MD Estoy muy en desacuerdo
BD Estoy bastante en desacuerdo
PD Estoy parcialmente en desacuerdo

Para facilitar el procedimiento e interpretación de datos se procedió a asignar una valoración cuantitativa a las distintas disposiciones del siguiente modo:

$\begin{array}{lll}\text { NC } & 0 & \text { No contesta } \\ \text { MA } & 1 & \\ \text { BA } & 2 & \\ \text { PA } & 3 & \\ \text { MD } & 4 & \\ \text { BD } & 5 & \\ \text { PD } & 6 & \end{array}$

Asimismo, con objeto de facilitar el análisis e interpretación de los resultados, los ítems se sometieron a categorización, por ejemplo el ítem 1, "Las personas con discapacidad con frecuencia son menos inteligentes que las demás personas", se denominaría "Inteligencia" (Anexo I).

El análisis factorial de la Escala puso de manifiesto la existencia de cinco factores que, en general, ratifican la estructura factorial de otros instrumentos similares desarrollados en otros países, como la Disability Factor Scale (Siller, 1967). El factor I, denominado Valoración de capacidades y limitaciones, comprende 10 ítems, aludiendo a la concepción que el encuestado tiene de las personas con discapacidad. El factor II, denominado Reconocimiento/Negación de derechos, aglutina 11 ítems y se refiere al reconocimiento de derechos fundamentales de la persona. El factor III, Implicación personal, está formado por juicios referidos a comportamientos concretos de interacción que la persona llevaría a efecto en relación con personas con discapacidad, agrupa 7 ítems. El factor IV, Calificación genérica (engloba 5 ítems), comprende las atribuciones globales y calificaciones generales que se efectúan acerca de rasgos presuntamente definitorios de la personalidad o conducta de las 
personas con discapacidad. El factor V, Asunción de roles, consiste en presunciones que el encuestado realiza sobre la concepción que de sí mismos tienen los discapacitados (engloba 4 ítems).

\section{Procedimiento}

Los centros universitarios participantes, como ya describimos anteriormente, fueron la Facultad de Ciencias de la Actividad Física y Deportes, Facultad de Psicología y Facultad de Ciencias de la Educación, centros en los que la presencia de universitarios con discapacidad representaba el 1,7\%,6,6\% y 16,7\%, respectivamente. A continuación se eligieron los grupos representativos.

Seguidamente se contactó con los profesores y profesoras de las diferentes titulaciones a los que se les solicitó permiso para aplicar la Escala de Actitudes hacia las personas con discapacidad. En las diferentes aplicaciones se explicaron a los participantes los objetivos de la investigación de forma que pudieran aportar los comentarios y sugerencias que estimaran convenientes, a la vez que se les pidió su colaboración de forma voluntaria.

\section{Resultados}

Para la estructuración, ordenación y análisis de los datos se utilizó el paquete de programas estadísticos SPSS en su versión 11.5 para Windows, que por su versatibilidad y fiabilidad de uso parece especialmente indicado para investigaciones de tipo descriptivo.

Para el análisis de los datos correspondientes partimos de la estructura de la escala en cinco factores:

I. Valoración de las capacidades y limitaciones.

II. Reconocimiento/Negación de derechos.

III. Implicación Personal.

IV. Calificación genérica.

V. Asunción de roles.

\section{Factor I. Valoración de las capacidades y limitaciones}

En líneas generales, se constató el grado de acuerdo entre los estudiantes de las distintas titulaciones seleccionadas en relación a su concepción de las personas con discapacidad, de su capacidad de aprendizaje y de desempeño, e inferencias sobre aptitudes orientadas a la ejecución de las tareas (tabla 2).

- Inteligencia. Los datos obtenidos muestran un relativo desacuerdo respecto de la consideración de las personas con discapacidad como menos inteligentes (puntuación media 5).

- Tipo de trabajo. Tampoco están a favor de que un trabajo sencillo y repetitivo fuese el más apropiado para personas con discapacidad (puntuación media 4). 
TABLA 2: Valoración de las capacidades y limitaciones.

\begin{tabular}{|l|c|c|}
\hline & Media & Desviación típica \\
\hline Inteligencia & 5 & 1 \\
\hline Tipo de trabajo & 4 & 1 \\
\hline Instrucciones & 5 & 1 \\
\hline Funcionamiento & 4 & 1 \\
\hline Aspiraciones & 5 & 1 \\
\hline Personalidad & 2 & 1 \\
\hline Profesionalidad & 2 & 1 \\
\hline Relaciones laborales & 2 & 1 \\
\hline Constancia & 5 & 1 \\
\hline Consciencia & 2 & 1 \\
\hline
\end{tabular}

- Instrucciones. Los universitarios encuestados opinaron que los discapacitados en el ámbito laboral, no se limitan a la recepción de instrucciones simples (puntuación media 5).

- Funcionamiento. En general, los estudiantes se presentaron en desacuerdo en la consideración de que las personas con discapacidad funcionan en muchos aspectos como niños (puntuación media 4).

- Aspiraciones. Los estudiantes universitarios se posicionaron en desacuerdo en relación a la afirmación de que de las personas con discapacidad no puede esperarse demasiado (puntuación media 5).

- Personalidad y Profesionalidad. Se observa la opinión generalizada de que las personas con discapacidad tienen una personalidad tan equilibrada como cualquier otra, lo que les permite ser profesionales en igualdad de competencias (puntuación media 2).

- Relaciones laborales. Existe un grado de acuerdo al considerar que en un contexto laboral los discapacitados se entienden sin dificultad con el resto de trabajadores (puntuación media 2).

- Constancia y Consciencia. Los universitarios encuestados expresaron su desacuerdo ante la afirmación de que las personas discapacitadas son poco constantes (puntuación media 5), admitiendo que puedan ser tan conscientes (puntuación media 2) como las personas que no poseen dicho padecimiento.

\section{Factor II. Reconocimiento/Negación de derechos}

En este apartado se analizaron las valoraciones en cuanto al reconocimiento de derechos fundamentales de la persona (por ejemplo igualdad de oportunidades, votar, acceder a créditos, etc.) y, en particular, el derecho que tiene a la normalización y a la integración social (tabla 3). 
TABLA 3: Reconocimiento / Negación de derechos.

\begin{tabular}{|l|c|c|}
\hline & Media & Desviación típica \\
\hline Convivencia & 6 & 1 \\
\hline Oportunidades de empleo & 2 & 1 \\
\hline Ocio y tiempo libre & 1 & 1 \\
\hline Relaciones personales & 2 & 1 \\
\hline Centros de educación especial & 5 & 2 \\
\hline Participación ciudadana & 6 & 1 \\
\hline Convivencia y trabajo & 1 & 1 \\
\hline Economía & 5 & 1 \\
\hline Consecución de metas & 1 & 1 \\
\hline Prevención y discapacidad & 5 & 1 \\
\hline Legislación & 6 & 1 \\
\hline
\end{tabular}

- Convivencia. Los universitarios expresaron un alto grado de desacuerdo, como reflejan las puntuaciones medias (5/6), en cuanto a la opinión de que las personas con discapacidad no deberían vivir con personas afectadas por el mismo problema.

- Oportunidades de empleo - Ocio y tiempo libre - Relaciones personales - Economía - Participación ciudadana. Se afirma que los discapacitados deberían tener las mismas posibilidades de acceso al mercado laboral, divertirse con las demás personas, casarse si lo desean, participar en votaciones, etc.

- Centros de educación especial. Los encuestados apostaron por la integración al afirmar que los discapacitados no deben ser recluidos en centros de educación especial (puntuación media 5).

- Convivencia y trabajo. Las puntuaciones obtenidas, media cercana a 1, permiten concluir que los estudiantes consideran apropiada la convivencia en el ámbito laboral y personal.

- Consecución de metas. Generalmente, los universitarios opinaron (medias 1/2) que las personas con discapacidad pueden llevar a cabo tareas con resultados análogos a los que no padecen dificultad.

- Prevención y discapacidad. Por último, se muestran bastante en desacuerdo en la cuestión relativa a la adopción de medidas preventivas sobre la maternidad de los que padecen déficit de diversa índole (puntuación media 5).

\section{Factor III. Implicación personal}

En este bloque se abordan los juicios referidos a comportamientos concretos de interacción en relación con los discapacitados. Se constata, en este sentido, que los estudiantes 
universitarios encuestados presentan una predisposición favorable a actuar y mostrar una aceptación efectiva de las personas con discapacidad en situaciones sociales, personales y laborales (tabla 4).

TABLA 4: Implicación personal.

\begin{tabular}{|l|c|c|}
\hline & Media & Desviación típica \\
\hline Relaciones sociales & 1 & 1 \\
\hline Aceptación de la diferencia & 5 & 2 \\
\hline Reclusión social & 6 & 1 \\
\hline Trabajo & 1 & 1 \\
\hline Trabajo y discapacidad & 6 & 1 \\
\hline Situaciones sociales & 6 & 1 \\
\hline Familia y discapacidad & 6 & 1 \\
\hline
\end{tabular}

- Relaciones Sociales - Situaciones sociales. Los encuestados asienten positivamente ante situaciones sociales que faciliten las relaciones sociales con los discapacitados.

- Aceptación de la diferencia - Reclusión social. Los estudiantes encuestados se muestran generalmente en desacuerdo ante la reclusión social de las personas que padecen discapacidad (media 5/6).

- Trabajo - Trabajo y discapacidad. A grandes rasgos, tal y como expresan las puntuaciones medias obtenidas, existe una aceptación positiva de las personas con discapacidad en situaciones laborales (puntuación media 1/6).

- Familia y discapacidad. Ante la cuestión relativa a "en el caso de tener un familiar con discapacidad, evitaría comentarlo con otra persona", los estudiantes de las diferentes titulaciones se mostraron en desacuerdo ante la ocultación de tal situación (media 6).

\section{Factor IV. Calificación genérica}

Recoge las atribuciones globales y calificaciones generales que los encuestados efectúan acerca de los rasgos presuntamente definitorios de la personalidad o conducta de las personas con discapacidad, no apreciándose valoraciones que denoten etiquetajes de tono estereotipado, negativo o peyorativo sobre las personas discapacitadas.

- Sentido del humor - Resentimiento. Las puntuaciones medias obtenidas a nivel global y diferencial en función de la titulación evidencian un desacuerdo relativo en el hecho de que las personas con discapacidad se encuentran siempre de mal humor o resentidas con las personas consideradas "físicamente normales" (puntuación media 5).

- Sociabilidad. Los universitarios encuestados consideran que las personas discapacitadas son bastante sociables (puntuación media 2).

- Suspicacia - Trabajo entre iguales. Los estudiantes universitarios encuestados se posicionaron muy en desacuerdo en relación a la consideración de que las personas con 
TABLA 5: Calificación genérica.

\begin{tabular}{|l|c|c|}
\hline & Media & Desviación típica \\
\hline Sentido del humor & 5 & 1 \\
\hline Sociabilidad & 2 & 1 \\
\hline Suspicacia & 4 & 2 \\
\hline Resentimiento & 5 & 1 \\
\hline Trabajo entre iguales & 4 & 1 \\
\hline
\end{tabular}

discapacidad generalmente son suspicaces (puntuación media 4). De un modo similar, los encuestados expondrían que los discapacitados en determinadas situaciones no tienen motivos para preferir trabajar con sus iguales (puntuación media 4).

\section{Factor V. Asunción de roles}

En este apartado se detallan las puntuaciones medias y desviaciones típicas obtenidas en relación a las presunciones que el encuestado efectúa acerca de la concepción que de sí mismas tienen las personas con discapacidad; apreciándose puntuaciones muy similares en las respuestas emitidas (tabla 6 ).

TABLA 6: Asunción de roles.

\begin{tabular}{|l|c|c|}
\hline & Media & Desviación típica \\
\hline Confianza en sí mismo & 3 & 1 \\
\hline Vida social & 2 & 1 \\
\hline Satisfacción personal & 3 & 1 \\
\hline Valoración personal & 3 & 1 \\
\hline
\end{tabular}

- Confianza en sí mismo - Satisfacción personal - Valoración personal. Los encuestados expusieron un acuerdo parcial, respecto de la consideración de que los que padecen déficit confían en sí mismos, se valoran y se sienten satisfechos consigo mismos (puntuación media 3).

- Vida social. Finalmente, existe un acuerdo prácticamente total entre aquellos estudiantes que cumplimentaron el cuestionario, respecto de que la discapacidad no es un impedimento para llevar una vida normal (puntuación media 2).

\section{Conclusiones}

En líneas generales, los datos obtenidos nos permiten concluir que existe un alto grado de acuerdo y apreciación positiva entre los participantes en relación a: su concepción de las 
personas con discapacidad, el reconocimiento de sus derechos fundamentales, su predisposición a actuar y mostrar una aceptación efectiva de las mismas; acuerdo sobre los rasgos que, presuntamente, definen la personalidad o conducta del discapacitado y las presunciones acerca de la satisfacción, valoración personal y confianza que en sí mismo tienen los discapacitados. Aunque los resultados concuerdan con los escasos trabajos realizados en esta línea por otros autores (Gómez e Infante, 2004; González, Gilar y Pérez, 2002; Diario Hoy de $2 / 2 / 05$ ), si se revisan estas investigaciones es necesario realizar una serie de puntualizaciones. Así el estudio realizado por Gómez e Infante (2004) se basa en el análisis de las actitudes de los estudiantes universitarios chilenos, cuyo contexto puede diferir de la población universitaria de nuestro medio; González, Gilar y Pérez (2002) se centraron en las actitudes de los estudiantes universitarios hacia la discapacidad analizando una única titulación, mientras que la investigación que nosotros presentamos y la realizada por López (Diario Hoy de 2/2/05) han abordado titulaciones diversas.

Los datos obtenidos en nuestro estudio, aunque creemos que son interesantes y se encuentran en la vía de los realizados por otros autores, deberían ser interpretados con cierta cautela, puesto que aluden a un porcentaje limitado de población universitaria granadina, en contacto con estudiantes con discapacidad que cursan similar o idéntica titulación. Si bien los datos son alentadores, debemos señalar que puede deberse a que los estudiantes con discapacidad sean muy competentes en el terreno académico, personal o social, provocando por ello actitudes positivas. De acuerdo con un estudio, llevado a cabo por las autoras de este trabajo, evaluando las barreras sociopersonales de 29 estudiantes universitarios granadinos con discapacidad física y/o sensorial, se encontró que éstos expresaban no sentir prejuicios por parte de sus compañeros, se sentían sociables, valorados por los demás en el trabajo que desempeñaban, encontraban con facilidad ayuda cuando lo requerían, no se sentían excluidos cuando debían trabajar en equipo; afirmaron sentirse igual que los demás, de acuerdo con su peculiaridad; y señalaban que la discapacidad no es un obstáculo para el mantenimiento de una relación familiar. Todos estos aspectos les permitían sentirse autorrealizados (Polo y López-Justicia, 2005).

Es evidente la importancia que tienen las actitudes positivas para el éxito en el proceso educativo (Reina, 2003), siendo necesario, por tanto, desarrollarlas y potenciarlas; ello implica la puesta en práctica de una labor de orientación que lleve a entender que la diversidad de la experiencia humana es una de las tareas fundamentales de la educación para el siglo XXI (Delors, 1996). La labor educadora y orientadora crece en calidad, pero también en complejidad, a partir del momento en que desea mostrarse respetuosa con la diferencia y cuando procura dar una respuesta educativa adecuada que contemple esa diferencia.

Desde hace unos años han proliferado los servicios de orientación en contextos universitarios con el fin de desarrollar acciones que lleven al logro de una sociedad más justa y equitativa con igualdad de oportunidades, que permitan clarificar muchos prejuicios y estereotipos que se encuentran constantemente; la educación viene a ser un vehículo ideal para llevarnos hacia este modelo social y la orientación un espacio, dentro de la educación para el análisis, la discusión y la toma de consciencia sobre lo que a cada cual le corresponde realizar y lo que en conjunto queremos construir y alcanzar (Ministerio de Educación Pública, 2005). En el presente trabajo se observa un cambio positivo, cuyo ejemplo lo constituye la población universitaria granadina, llevándonos a concluir que algunas de las acciones que vienen desarrollándose desde los Gabinetes de Atención Social 
de las Universidades, pueden ser acertadas (Polo y López-Justicia, 2004). Pero aún queda mucho por hacer hasta que la población universitaria tome conciencia de las necesidades y posibilidades de las personas discapacitadas, por eso creemos que además de acciones emprendidas como asesoramiento (para conocer las necesidades relacionados con el estudio de discapacitados para determinar apoyos), o accesibilidad (para adecuar el medio físico a las dificultades de los estudiantes con discapacidad, realizando adaptaciones arquitectónicas); deberían contemplarse otras tales como la sensibilización y formación para fomentar actitudes positivas hacia las personas con discapacidad en la comunidad universitaria, mediante la realización y colaboración en actividades formativas de intercambio de experiencias.

Los resultados del presente estudio hacen pensar que los estudiantes granadinos muestran actitudes positivas hacia la discapacidad, lo cual es muy gratificante porque parece que se avanza en el reconocimiento de que estas personas son capaces de contemplar opciones propias de vida tan legítimas como los demás integrantes de la sociedad; lo que supone un paso en la integración y participación en igualdad de condiciones y su pleno reconocimiento social. Al parecer, el cometido de los servicios de orientación surte algunos de los efectos deseados no solo entre la población afectada si no que se refleja en los demás estudiantes. A pesar de los datos favorables hallados en este estudio llamamos la atención sobre la conveniencia de diseñar otros con una muestra mayor (a ser posible ínter-universitaria) en la que se analicen los temas planteados en éste, así como comprobar aspectos como la posible relación entre actitudes positivas o negativas y el hecho de tener contacto o no con la discapacidad. Con frecuencia las actitudes no declaradas son las que determinan las posibilidades de integración de las personas discapacitadas y las actitudes y prejuicios frente a ellas tienen tal importancia y valor en el proceso de integración y socialización, que, como hemos puesto de manifiesto, han sido estudiadas desde diferentes puntos de vista. En el ámbito educativo hemos podido corroborar en el presente estudio que las actitudes hacia los alumnos con necesidades educativas especiales en el contexto universitario son, generalmente, bastante positivas lo que nos llevaría a la necesidad de conocer cuáles son las características de la persona y del contexto que favorecen las relaciones interpersonales entre alumnado, dadas las implicaciones que se derivan de esta situación.

\section{Referencias bibliográficas}

Alcantud, F. (1995). "Estudiantes con discapacidades integrados en los estudios universitarios. Notas para su integración". En F. Rivas, Manual de Asesoramiento y Orientación Vocacional (pp. 455470). Madrid: Síntesis.

Alemany, L. Ma . (2003). "La actitud del profesorado hacia la integración del alumnado con necesidades educativas especiales". Convergencia, Año 11, n 34, Enero-Abril 2004.

Colás, M $M^{a}$. P. (1994). "Los métodos descriptivos". En Ma. P. Colás y L. Buendía, Investigación educativa (pp. 177-199). Sevilla: Alfar.

Delors, J. (1996). La educación encierra un tesoro. Informe a la UNESCO de la Comisión Internacional para el siglo XXI. Madrid: Santillana.

Diario Hoy (2005, 2 de Febrero). "Las alumnas de la UEx tienen mejor actitud hacia los discapacitados que los hombres". Extraído el 10 de febrero de 2005 desde http:/ www.extremadura.com/general/noticias. 
Figuera, P. (1996). "La inserción del universitario en el mercado de trabajo". En P. Figuera, El marco de la inserción en el ámbito de la población universitaria (pp. 96-157). Barcelona: E.U.B.

Gómez, V. e Infante, M. (2004). "Actitudes de los estudiantes de educación hacia la integración de las personas con discapacidad y hacia la educación multicultural”. Cultura y Educación, vol 16 (4), 371-383.

González, M. C., Gilar, R. y Pérez, A. M. (2002). Perfil actitudinal del alumnado de magisterio hacia la discapacidad. Comunicación presentada en el VII Congreso Internacional "Exigencias de la Diversidad", Santiago de Compostela (pp. 46-46).

Larrivee, B. (1982) "Factors underlying regular classroom teachers attitude toward mainstreaming". Psychology in the Schools, 19, 374-379.

López-Justicia, Ma. D y Chacón, A. (2004). "Acceso a la educación de los alumnos y alumnas afectados de baja visión”. En $\mathrm{M}^{\mathrm{a}}$. A. López-Justicia, Aspectos evolutivos y educativos de la deficiencia visual (pp. 115-149). A Coruña: Netbiblo.

Ministerio de Educación Pública. República de Costa Rica. (2005, 1 de Diciembre). Programas de Estudio 2005. Orientación Educación Diversificada. Extraído el 10 de febrero de 2005 desde http://www.mep.go.cr/DescargasHTML/Curricular/doc/OrientacionIVCiclo.doc.

Polo, Ma . T. y López-Justicia, $M^{\mathrm{a}}$. D. (2004). "Análisis de los factores que influyen en el proceso de inserción sociolaboral de estudiantes con discapacidad de la Universidad de Granada". Revista de Enseñanza Universitaria, $\mathrm{n}^{\circ} 24, \mathrm{pp} .87-98$.

Polo, Ma . T. y López-Justicia, Ma . D. (2005). "Barreras de acceso al medio físico de los estudiantes con discapacidad motora de la Universidad de Granada". Revista Electrónica de Investigación Psicoeducativa, $\mathrm{n}^{\circ}$ 7, 3 (3), pp. 121-132.

Reina, R. (2003). "Propuesta de intervención para la mejora de actitudes hacia personas con discapacidad a través de actividades deportivas y recreativas". http: www.efdeportes.com/Revista digital. Buenos Aires, Año 9, n 59, Abril 2003.

Rizzo, T. L. y Wright, R. G. (1988). "Selected attributes related to physical educators' attitudes toward teaching students with handicaps". Mental Retardation, 26, pp. 307-309.

Siller, J. (1967). General form of the Disability Factor Scale (DFS-G). New York University. No publicado.

Triandis, H. C. (1971). Attitude and attitude change. Nueva York: Wiley.

Verdugo, M. A., Jenaro, C. y Arias, B. (1995). "Actitudes sociales y profesionales hacia las personas con discapacidad: Estrategias de evaluación e intervención". En M. A. Verdugo (dir.), Personas con discapacidad. Perspectivas psicopedagógicas y rehabilitadoras (pp. 79-135). Madrid: Siglo XXI Editores.

Zulueta, M. I. (1991). "Actitudes de los padres: Una aproximación psicológica". Síndrome de Down: Avances en acción familiar. Santander Cantabria. Fundación Síndrome de Down de Cantabria, 67-69.

Fecha de recepción: 27-04-05

Fecha de revisión: 15-02-06

Fecha de aceptación: 17-10-06 


\section{ANEXO}

1. Las personas con discapacidad con frecuencia son menos inteligentes Inteligencia

2. Un trabajo sencillo y repetitivo es el más apropiado para las personas con discapacidad Tipo de trabajo

3. Permitiría que mi hijo aceptase la invitación a un cumpleaños que le hiciera un niño con discapacidad Relaciones sociales

4. En el trabajo, una persona con discapacidad sólo es capaz de seguir instrucciones simples

Instrucciones

5. Me disgusta estar cerca de personas que parecen diferentes, o actúan de forma diferente Aceptación de la diferencia

6. Las personas con discapacidad deberían vivir con personas afectadas por el mismo problema Convivencia

7. Las personas con discapacidad funcionan en muchos aspectos como los niños Funcionamiento

8. De las personas con discapacidad no puede esperarse demasiado Aspiraciones

9. Las personas con discapacidad deberían tener las mismas oportunidades de empleo que cualquier otra persona

Oportunidades de empleo

10. Las personas con discapacidad deberían mantenerse apartadas de la sociedad Reclusión social

11. No me importaría trabajar junto a personas con discapacidad Trabajo

12. Las personas con discapacidad deberían poder divertirse con las demás personas Ocio y tiempo libre

13. Las personas con discapacidad tienen una personalidad tan equilibrada como cualquier otra persona

Personalidad

14. Las personas con discapacidad deberían poder casarse si lo desean Relaciones personales

15. Las personas con discapacidad deberían ser confinadas en instituciones especiales Centros de educación especial

16. Muchas personas con discapacidad pueden ser profesionales competentes Profesionalidad

17. A las personas con discapacidad se les deberían impedir votar Participación ciudadana 
18. Las personas con discapacidad a menudo están de mal humor Sentido del humor

19. Las personas con discapacidad confían en sí mismas tanto como las personas normales Confianza en sí mismo

20. Generalmente las personas con discapacidad son sociables Sociabilidad

21. En el trabajo, las personas con discapacidad se entienden sin problemas con el resto de los trabajadores

\section{Relaciones laborales}

22. Sería apropiado que las personas con discapacidad trabajaran y vivieran con personas normales Convivencia y trabajo

23. A las personas con discapacidad se les debería prohibir pedir créditos o préstamos Economía

24. Las personas con discapacidad generalmente son suspicaces

\section{Suspicacia}

25. No quiero trabajar con personas con discapacidad Trabajo y discapacidad

26. En situaciones sociales, preferiría no encontrarme con personas con discapacidad Situaciones sociales

27. Las personas con discapacidad pueden hacer muchas cosas tan bien como cualquier otra persona

Consecución de metas

28. La mayoría de las personas con discapacidad están resentidas con las personas físicamente normales

\section{Resentimiento}

29. La mayor parte de las personas con discapacidad son poco constantes Constancia

30. Las personas con discapacidad son capaces de llevar una vida social normal Vida social

31. Si tuviera un familiar cercano con discapacidad, evitaría comentarlo con otras personas Familia y discapacidad

32. La mayor parte de las personas con discapacidad están satisfechas de sí mismas

\section{Satisfacción personal}

33. La mayoría de las personas con discapacidad sienten que son tan valiosas como cualquiera Valoración personal

34. La mayoría de las personas con discapacidad prefieren trabajar con otras personas que tengan su mismo problema

Trabajo entre iguales

35. Se debería prevenir que las personas con discapacidad tuvieran hijos Prevención y discapacidad 
36. Las personas con discapacidad son en general tan conscientes como las personas normales

Consciencia

37. Deberían existir leyes que prohibieran casarse a las personas con discapacidad Legislación 